\title{
COMMISSION 16: PHYSICAL STUDY OF PLANETS AND SATELLITES (ÉTUDE PHYSIQUE DES PLANĖTES ET DES SATELLITES)
}

\author{
Report of Meetings, 23, 24 and 28 August 1973
}

President: G. H. Pettengill.

Three regular half-day sessions of the Commission were held with topics as listed below. In addition, the Commission sponsored (together with Commission 17) a Joint Discussion on the Origin of the Moon and Satellites, which took place on 29 August.

\section{General Business Session, 23 August 1973}

The Working Group on Martian Nomenclature, chaired by G. de Vaucouleurs, presented its final draft report and recommendations. These will be published in full in a forthcoming issue of the journal Icarus, since they are too lengthy for inclusion here. The draft was approved by the Commission members present. An abbreviated version describing the general scheme of the recommendations is given below, followed by two adopted resolutions concerned with coordinate systems.

\section{RECOMMENDATIONS OF THE WORKING GROUP ON MARTIAN NOMENCLATURE}

\section{Designation of Provinces}

Instead of the original concept of 'provinces' defined by classical albedo features, which proved unworkable in practice because of the variability of many of these markings and the frequent indefiniteness of their boundaries, we propose to divide the surface of the planet into 30 geometric areas. The equatorial areas between $+30^{\circ}$ and $-30^{\circ}$ latitude are covered by 16 maps in Mercator projection measuring $45^{\circ}$ in longitude by $30^{\circ}$ in latitude. The intermediate latitudes between $\pm 30^{\circ}$ and $\pm 65^{\circ}$ are covered by 12 maps in Lambert conformal projection each measuring $60^{\circ}$ in longitude by $35^{\circ}$ in latitude; the polar regions between $\pm 65^{\circ}$ and $\pm 90^{\circ}$ are covered by two maps in polar stereographic projection.

Each chart is designated by the name of a prominent classical albedo feature within its confines and by a 3-letter abbreviation which will be used as a prefix to the crater designation scheme described below (Figure 1).

\section{Crater Designations}

Within each chart all craters larger than approximately $20 \mathrm{~km}$ are assigned a two-letter designation from $\mathrm{Aa}$ to $\mathrm{Za}$ in which the first letter is alphabetically in order of increasing longitude from east to west and the second in order of increasing latitude from south to north. A total of approximately 6000 craters have been so designated and are marked on the charts of the Atlas of Topographic Maps prepared at the U.S. Geological Survey (available to Commission 16 members on request). In a few heavily cratered areas the capacity of the two-letter scheme is exceeded and a third letter is added to provide a designation for all craters larger than the $20 \mathrm{~km}$ limit. It is recommended that Martian craters be identified by the chart prefix followed by the letter designation (e.g. SYR Aa). 


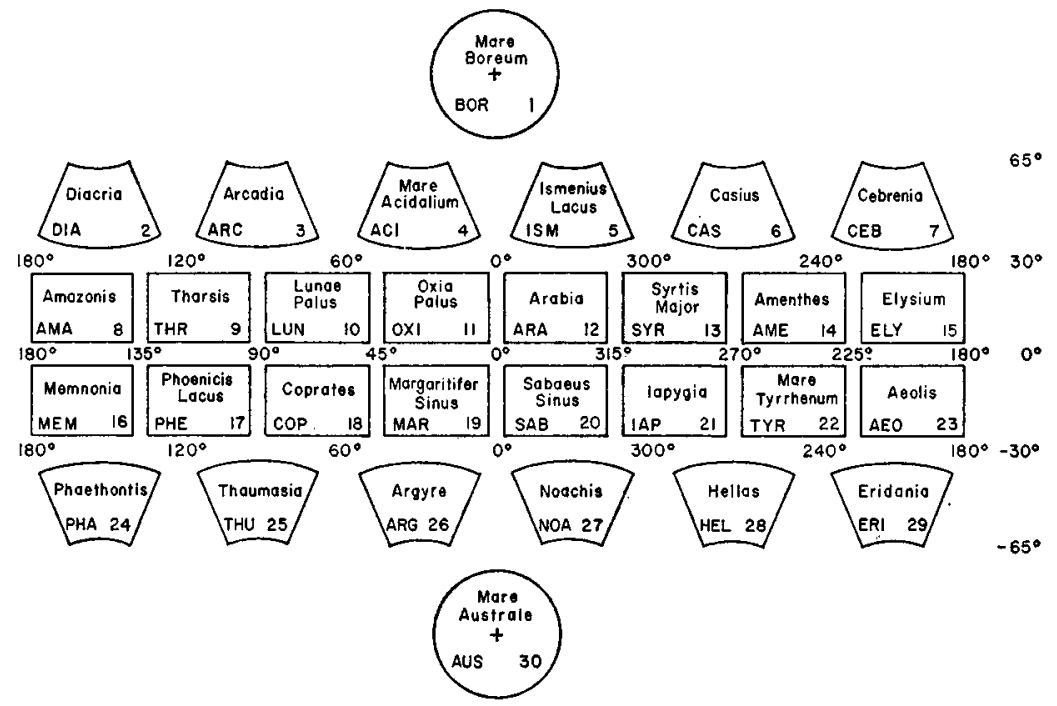

Fig. 1. Mars Map quadrangles designations and 3-letter abbreviations.

\section{Named Craters}

In addition to the letter scheme, a selection of the largest craters, generally larger than $100 \mathrm{~km}$ in diameter, receive names of prominent, deceased individuals who have contributed either to the scientific study of Mars, or the lore of the planet, or to basic discoveries of significance to the exploration of Mars or the interpretation of its phenomena. After extensive discussions a consensus list of about 180 names was selected.

\section{Other Topographic Features}

Thirteen classes of topographic features are designated below. Features in twelve of these classes receive specific names adopted from nearby classical albedo features taken from the Schiaparelli or Antoniadi maps. Usually following the name is the class of feature, e.g. Olympus Mons. One class of features, the valleys, is treated differently and named after 'MARS' in various languages.

Name of Class
Catena
Chasma
Dorsum (Dorsa)
Fossa (Fossae)
Labyrinthus
Mensa (Mensae)
Mons (Montes)
Patera
Planitia
Planum
Tholus
Vallis (Valles)
Vastitas

Name of Class

Catena

Fossa (Fossae)

Labyrinthus

Mensa (Mensae)

Patera

Planitia

Vastitas

\section{Description of Feature}

chain of craters

canyon

ridge(s)

long, narrow, straight or curved valley (s)

intersecting valley complex

mesa; flat-topped elevation (s)

mountain(s)

shallow complex crater with scalloped edges

low plain

plateau or high plain

small domical mountain or hill

sinuous valley (s)

widespread lowlands 


\section{Craters on Satellites}

Craters on Phobos are named after those involved with the discovery, dynamics, or properties of the Martian satellites and who are not already commemorated on Mars. Craters on Deimos are named after authors of literary or artistic allusions to the Martian satellites.

\section{RESOLUTION ON THE PHYSICAL EPHEMERIS OF MARS}

(Adopted by Commissions 4 and 16)

Considering that recent new determinations of the rotational elements of Mars indicate the need for a revision of the elements currently adopted in the physical ephemeris of Mars, and that a new approach to the definition of the origin of areographic longitudes appears useful (G. de Vaucouleurs, M. E. Davies and F. M. Sturms, Jr, J. Geophys. Res. 78, 4395, 1973), Commissions 4 and 16 recommend

(1) that new elements and a new definition of the origin of the areographic longitudes, as given below, be incorporated in the physical ephemeris of Mars after 1979 or as soon as deemed practicable in the judgment of the cognizant Directors of the National Ephemeris Offices, but not before the tie between the new and current systems has been firmly established by appropriate comparisons between the ground-based and Mariner 9 coordinate systems.

The new elements are as follows:

(a) The R.A. and $\mathrm{D}$ of the North Pole of Mars (referred to the standard equinox and equator of 1950.0 ) are given by

$$
\begin{aligned}
& \alpha_{0}=317.32-0.1011 T \\
& \delta_{0}=+52.68-0.0570 T,
\end{aligned}
$$

where $T$ is measured in Julian centuries of 36525 days from JD 2433282.5.

(b) The angle measured in the direct sense along the Mars equator from its ascending node on the standard equator of 1950.0 to the zero point of areocentric RA is given by

$$
\phi_{0}=222.93538-0.09040 T-0.00010 T^{2}
$$

(c) The areocentric RA of the prime meridian of Mars is given by

$$
V=V_{\mathrm{o}}+350.892017(\mathrm{JD}-2433282.5) \text {. }
$$

The Julian Date is reckoned in ephemeris time. The provisionally adopted value of $V_{0}$ is $148: 24$. The rotation rate results from a new analysis of the observations of the past century (de Vaucouleurs et al., loc. cit.).

The following notes on the preceding statement of the elements are intended for explanatory purposes only.

(i) the linear terms in the expression for $\alpha_{0}$ and $\delta_{0}$ correspond to a precession of the axis of rotation about the pole of the orbit at the rate of 0.0837 per century.

(ii) the corresponding inclination of the equator to the orbital plane of Mars is given by

$$
I=25: 19969+0.01219 T+0 \% 00006 T^{2}
$$

(iii) the zero point of areocentric right ascension corresponds to the vernal equinox (i.e. the ascending node of the orbital plane on the equator of Mars).

(iv) the adopted prime meridian is intended to be such that the center of the small crater Airy- 0 has zero areographic longitude.

(2) that the present investigations be continued in order to establish precisely the tie between the new and the current systems, and

(3) that a recomputation of the physical ephemeris based on these new elements be prepared for the years 1800 to 1979 . 


\section{RESOLUTION ON SATELLITE COORDINATE SYSTEMS}

For all satellites except the Moon, the origin of longitude is defined by the sub-planetary intersection of the satellite's equator and the plane containing the center of the satellite, the planet and the Sun at the first superior heliocentric conjunction of the satellite after 1950.0. The conventions for the direction of increasing longitude and for the choice of north pole shall be the same as adopted for the planets at the IAU 14th General Assembly in 1970.

\section{WORKING GROUP FOR PLANETARY AND SATELLITE NOMENCLATURE}

The accelerating rate of planetary exploration by spacecraft has created the need for a more rapid procedure for naming surface features than is possible in the present approach, tied to triennial IAU General Assemblies. To this end, the IAU Executive Committee has created an InterCommission Working Group for Planetary and Satellite Nomenclature, with considerable power to act in the name of the Committee between Assemblies. The Chairman of the Group is Peter Millman; other members include the presidents of Commissions 16 and 17 and the Heads of 5 Panels. The latter are charged with the responsibility for naming features on the Moon, Mercury, Venus, Mars and the Galilean satellites of Jupiter. Recommendations are originated in the Panels, passed to the main Group for consideration and possible revision, then sent to the Executive Committee for approval. The actions of this group in the preceding 3 years will be reviewed, of course, at each General Assembly.

\section{SCIENTIFIC PRIORITIES FOR PLANETARY RESEARCH}

Commission 16 unanimously voted in this session to refrain from the setting of scientific priorities. It was felt that a list of such priorities would serve no useful purpose and might, in fact, lead to embarrassment if misunderstood by national funding agencies.

\section{Spacecraft Results and Plans, 24 August 1973}

Titles of presentations:

C. Sagan: Variable Features and Climatic Cycles on Mars.

H. Masursky: Fixed Surface Features and Geology of the Martian Surface.

B. A. Smith: Experimental Objectives of the U.S. Mariner Venus/Mercury and Mariner Jupiter/ Saturn Missions.

J. S. Hall: Report on the Lowell Observatory.

\section{Scientific Papers, 28 August 1973}

Titles of presentations:

D. B. Campbell: Topography and Other Radar Results for Mercury and Venus.

S. K. Runcorn: The Shape and Internal Dynamics of Mars.

J. R. Dickel: Jupiter's Radiation Belts and Upper Atmosphere.

A. Dollfus: Cartography of the Galilean Satellites.

S. K. Runcorn: The Rotation of Jupiter.

A. F. Cook: The Rings of Saturn.

C. Sagan: The Atmosphere and Surface of Titan. 